Pacific Journal of Mathematics

AN ALGORITHM IN A COMPLEX FIELD AND ITS
APPLICATION TO THE CALCULATION OF UNITS 


\title{
AN ALGORITHM IN A COMPLEX FIELD AND ITS APPLICATION TO THE CALCULATION OF UNITS
}

\author{
Malvina Baica
}

\begin{abstract}
In the present paper the author develops a new periodic algorithm for certain algebraic number fields and calculates units in these fields from its periodicity. This algorithm (abbr. ACF) is a modification of the Jacobi-Perron Algorithm, where for the first time the expanding of the complex (not only real) numbers was possible. The ACF discloses new units of which the already known ones are special cases, and it has the advantage that many results in the theory of units can be derived by means of a unified periodic algorithm.
\end{abstract}

0. Introduction. Hilbert's dream and advice (Zahlbericht) of tailoring a universal algorithm by means of which the expansion of any real algebraic number (or any vector in $E^{n-1}, n \geq 2$ ) becomes periodic, thus enabling a complete system of fundamental units in the corresponding field to be calculated, still is, and probably will remain, far away from realization. Though C.G.J. Jacobi invented an algorithm for totally real cubic fields, generalizing ingeniously the Euclidean algorithm, he could not prove or disprove the periodicity of his new tool nor calculate units by its means. Jacobi's algorithm was later generalized by Perron [11] for any total real algebraic field, but even he, the master of continued fractions, did not succeed in proving or disproving the periodicity of his new algorithm.

In his last scientific work Perron [12], though not successfully, shows his efforts to solve the periodicity question of his algorithm. A partial answer to this question in the case of cubic irrationalities gave E. Dubois and R. Paysant-Le-Roux [6].

The title algorithm (abbr. ACF) can be interpreted as a modification of Jacobi-Perron Algorithm (abbr. JPA), where for the first time the employment of the complex (not only real) numbers was possible. The choice of $D_{i}-s$ to be complex numbers implies also a slight modification of the $T$-function in the companion vector.

This new algorithm ACF solves the problem of periodicity for infinitely many classes of algebraic numbers (but not for all of them) of any degree, and states explicitly some units in the corresponding fields. The periodicity of real algebraic numbers of degree $n \geq 3$ was first proved by Bernstein [3] for the JPA, and later for its modification $[3,1]$. 
The ACF discloses new units of which the already known ones by Bernstein [3], Halter-Koch [7], Halter-Koch and Stender [8] are special cases. The latter were obtained by the last two authors without an algorithm. This new algorithm ACF has the advantage that many results in the theory of units can be derived by means of a unified algorithm. The new units were also discovered by Neubrand [9], again without using an algorithm. Neubrand's method is quite different from the other methods, namely algebraic geometric or function theoretic oriented.

1. The ACF-definition, basic properties. We denote:

$$
S_{n+1}=\left\{d, D_{1}, D_{2}, \ldots, D_{n}\right\}, \quad n \geq 2,
$$

a set of any $n+1$ algebraic numbers;

$$
\mathscr{K}_{1}=Q\left(d, D_{1}, D_{2}, \ldots, D_{n}\right)
$$

the field generated by adjoining $S_{n+1}$ to $Q$;

$$
\left\{\begin{array}{l}
G(x)=\sum_{i=0}^{n} c_{i} x^{n-i} ; \quad c_{0}=1 ; c_{\imath} \in \mathscr{K}_{1}, i=1, \ldots, n \\
\text { an irreducible polynomial in } x \text { over } \mathscr{K}_{1} ;
\end{array}\right.
$$

$$
\left\{\begin{array}{l}
\mathscr{K}_{2}=\mathcal{K}_{1}(w) ; G(w)=0 \\
\text { the field generated by a root of } G(x) \text { in } \\
\text { adjunction with } \mathcal{K}_{1} .
\end{array}\right.
$$

Thus $\mathscr{K}_{2}=Q\left(w, d, D_{1}, D_{2}, \ldots, D_{n}\right)$ is of degree $n$ over $\mathcal{K}_{1}$ and of degree $n \cdot\left[\mathcal{K}_{1}: Q\right]$ over $Q$.

Definition 1. A vector $a^{(0)}$ in $\mathcal{K}_{2}^{n-1}(n \geq 2)$ with components which are functions of $w$ is called a starting or fixed vector; the vectors $a^{(v)} \in \mathcal{K}_{2}^{n-1}$ obtained by an algorithm from $a^{(0)}$,

$$
a^{(v)}=\left(a_{1}^{(v)}(w), a_{2}^{(v)}(w), \ldots, a_{n-1}^{(v)}(w)\right), \quad v=0,1, \ldots
$$

are called the current vectors; the vectors

$$
\left\{\begin{array}{l}
b^{(v)}=\left(b_{1}^{(v)}, b_{2}^{(v)}, \ldots, b_{n-1}^{(v)}\right) \in \mathcal{K}_{1}^{n-1}, \text { derived from } \\
a^{(v)} \text { or given by any formula are called the } \\
\text { companion vectors of the } a^{(v)}
\end{array}\right.
$$

We shall introduce the notation

$$
a_{0}^{(v)}, b_{0}^{(v)}=1, \quad v=0,1, \ldots
$$


Definition 2. The ACF of a starting vector $a^{(0)}$ is defined by the recurrence formula

$$
\begin{gathered}
a^{(v+1)}=\left(a_{1}^{(v)}-b_{1}^{(v)}\right)^{-1}\left(a_{2}^{(v)}-b_{2}^{(v)}, \ldots, a_{n-1}^{(v)}-b_{n-1}^{(v)}, 1\right) \\
a_{1}^{(v)}-b_{1}^{(v)} \neq 0 ; \quad v=0,1, \ldots
\end{gathered}
$$

From (1.8) we obtain

$$
a_{l}^{(v)}=b_{i}^{(v)}+\frac{a_{i-1}^{(v+1)}}{a_{n-1}^{(v+1)}} ; \quad i=1, \ldots, n-1 ; v=0,1, \ldots .
$$

We define the matrix entries

$$
\left\{\begin{array}{l}
A_{i}^{(j)}=\delta_{j}^{i} ; \quad i, j=0,1, \ldots, n-1, \delta_{j}^{i} \text { the Kronecker delta } \\
A_{i}^{(v+n)}=\sum_{j=0}^{n-1} b_{j}^{(v)} A_{i}^{(v+J)}, \quad i=0,1, \ldots, n-1 ; v=0,1, \ldots
\end{array}\right.
$$

From (1.10) we obtain easily by induction

$$
\left|\begin{array}{cccc}
A_{0}^{(v)} & A_{0}^{(v+1)} & \cdots & A_{0}^{(v+n-1)} \\
A_{1}^{(v)} & A_{1}^{(v+1)} & \cdots & A_{1}^{(v+n-1)} \\
\cdots \cdots & \cdots & \cdots & \cdots \\
A_{n-1}^{(v)} & A_{n-1}^{(v+1)} & \cdots & A_{n-1}^{(v+n-1)}
\end{array}\right|=(-1)^{v(n-1)}
$$

for $v=0,1, \ldots$

We obtain formula

$$
a_{i}^{(0)}=\frac{\sum_{j=0}^{n-1} a_{j}^{(v)} A_{i}^{(v+j)}}{\sum_{J=0}^{n-1} a_{j}^{(v)} A_{0}^{(v+j)}}, \quad i=1, \ldots, n-1 ; v=0,1, \ldots ;
$$

(1.12) is correct for $v=0$, giving on the right side,

$$
\left(0+0+\cdots+a_{i}^{(0)} A_{i}^{(i)}+0+\cdots+0\right) / A_{0}^{(0)}=a_{i}^{(0)} .
$$

Then substitute inductively on the right the values of $a_{j}^{(v)}$ from (1.10), (1.9).

We shall need the formula

$$
\prod_{j=1}^{v} a_{n-1}^{(j)}=\sum_{i=0}^{n-1} a_{i}^{(v)} A_{0}^{(v+i)}, \quad v=1,2, \ldots
$$

Proof by induction. (1.13) is correct for $v=1$. Then proceed as in the proof of (1.12). 
We shall need the basic formula

$$
\left\{\begin{array}{c}
\left|\begin{array}{ccccc}
1 & A_{0}^{(v+1)} & A_{0}^{(v+2)} & \cdots & A_{0}^{(v+n-1)} \\
a_{1}^{(0)} & A_{1}^{(v+1)} & A_{1}^{(v+2)} & \cdots & A_{1}^{(v+n-1)} \\
\vdots & \vdots & \vdots & & \vdots \\
a_{n-1}^{(0)} & A_{n-1}^{(v+1)} & A_{n-1}^{(v+2)} & \cdots & A_{n-1}^{(v+n-1)}
\end{array}\right| \\
=(-1)^{v(n-1)}\left(\sum_{j=0}^{n-1} a_{j}^{(v)} A_{0}^{(v+j)}\right)^{-1} .
\end{array}\right.
$$

Proof. Start with (1.11). Multiply both sides by $\sum_{j=0}^{n-1} a_{j}^{(v)} A_{0}^{(v+j)}$, add to the first column the $a_{j}^{(v)}$ multiple of the $j+1$ st column. The result is obtained by induction.

2. A periodic $\mathbf{A C F}$ - notations. In this section we begin preparation for the periodicity of the most general $a^{(0)} \in \mathscr{K}_{2}$. This $a^{(0)}$ will be specified later when we approach the central theorem. The choice of $a^{(0)}$ will result from specification of the numbers $d, D_{1}, D_{2}, \ldots, D_{n} \in S_{n+1}$ and the function $G(x)$ from (1.3). We first choose

$$
G_{F}(x)=-d+\prod_{j=1}^{n}\left(x-D_{\jmath}\right), \quad n \geq 2,
$$

where $G_{F}(x)$ is irreducible over $\mathcal{K}_{1}$. This polynomial was treated by Stender in his dissertation, if the $D_{i}$ are natural numbers.

$$
\left\{\begin{array}{l}
G_{F}\left(w_{i}\right)=0, i=1, \ldots, n ; \text { Let } w_{1}, w_{2}, \ldots, w_{n} \text { be the roots } \\
\text { of } G_{F}(x) \text { is an algebraic closure of } \mathscr{K}_{2}\left(w_{1}, \ldots, w_{n}\right) . \\
w \in\left\{w_{1}, w_{2}, \ldots, w_{n}\right\} .
\end{array}\right.
$$

Thus $w$ is chosen to be one fixed root of the $n$-roots of $G_{F}$. We introduce the notation

$$
\left\{\begin{array}{l}
f_{i, l}=f_{i}=w-D_{i}, \quad i=1,2, \ldots, n \\
f_{i, k}=\prod_{j=i}^{k}\left(w-D_{j}\right) ; \quad 1 \leq i \leq k \leq n
\end{array}\right.
$$

In $\mathcal{K}_{2}$ we have of course

$$
f_{i, k}=f_{i, k}(w), \quad 1 \leq i \leq k \leq n
$$


and need not add $f_{i, k}=f\left(w, D_{i}, \ldots, D_{k}\right)$. We have, from $(2.1)-(2.3)$

$$
\prod_{j=1}^{n}\left(w-D_{j}\right)=f_{1, n}=f_{1, n}(w)=d \text {. }
$$

The following operations will be useful

$$
\left\{\begin{array}{l}
\frac{1}{f_{i, k}(w)}=\frac{1}{f_{i, k}}=\frac{f_{1, i-1} f_{k+1, n}}{d} ; \quad i \geq 2, k \leq n-1, \\
\frac{1}{f_{1, k}}=\frac{f_{k+1, n}}{d}, \quad k \leq n-1 ; \quad \frac{1}{f_{l, n}}=\frac{f_{1, i-1}}{d}, \quad 2 \leq i \leq n .
\end{array}\right.
$$

For the fixed vector $a^{(0)}$ we now choose

$$
a^{(0)}=\left(f_{1, n-1}, f_{1, n-2}, \ldots, f_{1,2}, f_{2,2}\right) .
$$

We shall in the sequel conduct the ACF of $a^{(0)}$ from (2.7), getting the current vectors $a^{(v)}, v=1,2, \ldots$ The companion vectors of the current vectors are derived from the current vectors by the formula, remembering (2.4);

$$
\begin{cases}b_{s}^{(v)}=a_{s}^{(v)}\left(D_{1}\right) ; & s=1, \ldots, n-1 ; \\ b^{(v)} \in \mathcal{K}_{1}^{n-1}, & v=0,1, \ldots\end{cases}
$$

The choice of $D_{1} \in S_{n+1}$ for the derivation of $b^{(v)}$ from $a^{(v)}$ is, as we shall later see, completely arbitrary. Any $D_{i} \in S_{n+1}, i=1, \ldots, n$, would do.

The reader should pay priority-attention to the formula

$$
\left\{\begin{array}{l}
b_{s}^{(0)}=f_{1, n-s}\left(D_{1}\right)=\left[\left(w-D_{1}\right) \cdots\left(w-D_{n-s}\right)\right]_{w=D_{1}}=0 \\
s=1, \ldots, n-1
\end{array}\right.
$$

We shall illustrate the first step in the ACF of $a^{(0)}$, working out all the necessary details of (1.8). In the sequel the current vectors' result will sometimes be enumerated directly without going into the details. We obtain from (2.7), in view of (2.9)

$$
\left\{\begin{array}{l}
b_{i}^{(0)}=f_{1, n-i}\left(D_{1}\right)=0, \quad i=1,2, \ldots, n-2 ; \\
b_{n-1}^{(0)}=f_{2,2}\left(D_{1}\right)=f_{2}\left(D_{1}\right)=D_{1}-D_{2} .
\end{array}\right.
$$

Thus

$$
\begin{gathered}
b^{(0)}=\left(0,0, \ldots, 0, D_{1}-D_{2}\right) . \\
a_{1}^{(0)}-b_{1}^{(0)}=f_{1, n-1}-0=f_{1, n-1},
\end{gathered}
$$


and by (2.6)

$$
\begin{gathered}
\left(a_{1}^{(0)}-b_{1}^{(0)}\right)^{-1}=\frac{1}{f_{1, n-1}}=\frac{f_{n, n}}{d}=\frac{f_{n}}{d}, \\
a_{n-1}^{(0)}-b_{n-1}^{(0)}=f_{2,2}-\left(D_{1}-D_{2}\right)=w-D_{2}-\left(D_{1}-D_{2}\right)=w-D_{1}=f_{1}, \\
a_{i}^{(0)}-b_{i}^{(0)}=a_{l}^{(0)}-0=a_{l}=f_{1, n-1}, \quad i=2, \ldots, n-2
\end{gathered}
$$

and from (1.8);

$$
\begin{gathered}
a^{(1)}=d^{-1} f_{n}\left(f_{1, n-2}, f_{1, n-3}, \ldots, f_{1,2}, f_{1}, 1\right), \\
a^{(1)}=\left(d^{-1} f_{1, n-2} f_{n}, d^{-1} f_{1, n-3} f_{n}, \ldots, d^{-1} f_{1} f_{n}, d^{-1} f_{n}\right) .
\end{gathered}
$$

\section{The first fugue of the ACF of $a^{(0)}$.}

Definition 3. A sequence of $n-1$ current vectors (including the fixed vector), viz.

$$
a^{((n-1) v+j),} \quad v=0,1, \ldots ; j=0,1, \ldots, n-2,
$$

is called the $v+1$ st fugue (of the current vector) of the $\mathrm{ACF}$ of $a^{(0)}$; the sequence of the corresponding companion vectors-the $v+1$ st fugue of the companion vectors of the ACF of $a^{(0)}$, is

$$
b^{(n-1) v+J}, \quad v=0,1, \ldots ; j=0,1, \ldots, n-2 .
$$

From (2.11) we obtain

$$
\begin{gathered}
b^{(1)}=\left(0,0, \ldots, 0, d^{-1}\left(D_{1}-D_{n}\right)\right), \\
a_{1}^{(1)}-b_{1}^{(1)}=d^{-1} f_{1, n-2} f_{n}-0 ; \quad\left(a_{1}^{(1)}-b_{1}^{(1)}\right)^{-1}=f_{n-1}, \\
a_{n-1}^{(1)}-b_{n-1}^{(1)}=d^{-1} f_{n}-d^{-1}\left(D_{1}-D_{n}\right)=d^{-1} f_{1}, \\
a^{(2)}=f_{n-1}\left(d^{-1} f_{1, n-3} f_{n}, \ldots, d^{-1} f_{1,2} f_{n}, d^{-1} f_{1} f_{n}, d^{-1} f_{1}, 1\right), \\
a^{(2)}=\left(d^{-1} f_{1, n-3} f_{n-1, n}, \ldots, d^{-1} f_{1,2} f_{n-1, n}, d^{-1} f_{1} f_{n-1, n},\right. \\
\left.\quad d^{-1} f_{1} f_{n-1}, f_{n-1}\right) .
\end{gathered}
$$

We can now prove the important

LEMMA 1. The $i+1$ st current vector of the first fugue has the form

$$
\left\{\begin{array}{c}
a^{(l)}=\left(d^{-1} f_{1, n-l-1} f_{n-1+1, n}, d^{-1} f_{1, n-\imath-2} f_{n-\imath+1, n}, \ldots,\right. \\
\quad d^{-1} f_{1} f_{n-\imath+1, n}, d^{-1} f_{1} f_{n-i+1, n-1}, f_{1} f_{n-\imath+1, n-2}, \ldots, \\
\left.\quad f_{1} f_{n-\imath+1}, f_{n-i+1}\right) \\
i=2, \ldots, n-2 ; n \geq 4 .
\end{array}\right.
$$


Proof. (3.5) is correct for $i=2$, as can be verified from (3.4). By the method applied in calculating $a^{(1)}, a^{(2)}$, the reader will now have no difficulty in proving Lemma 1 by induction. The special cases $n=2,3$ will be observed separately because of their importance.

$$
\begin{array}{ll}
a^{(0)}=w-D_{2} ; & b^{(0)}=D_{1}-D_{2} \\
a^{(1)}=d^{-1}\left(w-D_{2}\right) ; & b^{(1)}=d^{-1}\left(D_{1}-D_{2}\right) \\
a^{(2)}=w-D_{2}=a^{(0)} . &
\end{array}
$$

Here we have a purely periodic continued fraction representation.

$$
\begin{aligned}
& \left(w-D_{1}\right)\left(w-D_{2}\right)-d=0 ; \quad w-D_{2}=\left[\overline{D_{1}-D_{2}}\right], \quad d=1 . \\
& w-D_{2}=\left[\overline{D_{1}-D_{2}, d^{-1}\left(D_{1}-D_{2}\right)}\right] ; \quad d>1 ; \quad d \mid D_{1}-D_{2} .
\end{aligned}
$$

For $n=3$, we obtain, $d \neq 1$.

$$
\begin{array}{ll}
a^{(0)}=\left(\left(w-D_{1}\right)\left(w-D_{2}\right), w-D_{2}\right) ; & b^{(0)}=\left(0, D_{1}-D_{2}\right) ; \\
a^{(1)}=\left(d^{-1}\left(w-D_{1}\right)\left(w-D_{3}\right), d^{-1}\left(w-D_{3}\right)\right) ; & b^{(1)}=\left(0, d^{-1}\left(D_{1}-D_{3}\right)\right) ; \\
a^{(2)}=\left(d^{-1}\left(w-D_{1}\right)\left(w-D_{2}\right), w-D_{2}\right) ; & b^{(2)}=\left(0, D_{1}-D_{2}\right) ; \\
a^{(3)}=\left(\left(w-D_{1}\right)\left(w-D_{3}\right), w-D_{3}\right) ; & b^{(3)}=\left(0, D_{1}-D_{3}\right) ; \\
a^{(4)}=\left(d^{-1}\left(w-D_{1}\right)\left(w-D_{2}\right), d^{-1}\left(w-D_{2}\right)\right) ; & b^{(4)}=\left(0, d^{-1}\left(D_{1}-D_{2}\right)\right) ; \\
a^{(5)}=\left(d^{-1}\left(w-D_{1}\right)\left(w-D_{3}\right), w-D_{3}\right) ; & b^{(5)}=\left(0, D_{1}-D_{3}\right) ; \\
a^{(6)}=\left(\left(w-D_{1}\right)\left(w-D_{2}\right), w-D_{2}\right)=a^{(0)} . &
\end{array}
$$

The ACF of $a^{(0)}$ is purely periodic, and the length of its primitive period is $m=6$.

For $d=1$ we obtain

$$
\begin{aligned}
& a^{(0)}=\left(\left(w-D_{1}\right)\left(w-D_{2}\right), w-D_{2}\right) ; \quad b^{(0)}=\left(0, D_{1}-D_{2}\right) ; \\
& a^{(1)}=\left(\left(w-D_{1}\right)\left(w-D_{3}\right), w-D_{3}\right) ; \quad b^{(1)}=\left(0, D_{1}-D_{3}\right) . \\
& a^{(2)}=a^{(0)} ; \quad\left(w-D_{1}\right)\left(w-D_{2}\right)\left(w-D_{3}\right)-d=0, \quad \text { generally. }
\end{aligned}
$$

As a numerical example in the case $n=2$;

$$
\begin{gathered}
(w-4)(w-2)-2=0 ; \quad \text { choose } w=3+\sqrt{3} ; \quad D_{1}=4 ; \\
D_{2}=2 ; \quad d=2 . \quad \mathcal{K}_{1}=Q ; \quad \mathcal{K}_{2}=Q(\sqrt{3}) ; \\
a^{(0)}=w-2=[\overline{4-2,1 / 2(4-2)}] \\
\sqrt{3}+3-2=[2,1] ; \quad \sqrt{3}=[1, \overline{1,2}],
\end{gathered}
$$

a very simple way of constructing the periodic continued fraction of $\sqrt{3}$. 
4. The first fugue of the ACF of $a^{(0)}$, continued. From (2.7), (2.11) and (3.5) we have completed the calculations of the first fugue of the ACF of $a^{(0)}$, viz.

$$
\left\langle a^{(0)}, a^{(1)}, \ldots, a^{(l)}, \ldots, a^{(n-2)}\right\rangle, \quad i=2, \ldots, n-2
$$

altogether the fixed vector $a^{(0)}$, followed by $n-2$ current vectors. We obtain for the $n-1$ st vector, substituting $i=n-2$ in (3.5),

$$
a^{(n-2)}=\left(d^{-1} f_{1,1} f_{3, n}, d^{-1} f_{1} f_{3, n-1}, f_{1} f_{3, n-2}, \ldots, f_{1} f_{3}, f_{3}\right) .
$$

From (4.1) we obtain, calculating (in detail because of its essential pattern) the next current vector $a^{(n-1)}$. For this purpose we have

$$
b^{(n-2)}=\left(0,0,0, \ldots, 0, D_{1}-D_{3}\right)
$$

and from (4.1), (4.2)

$$
\left\{\begin{array}{l}
a_{1}^{(n-2)}-b_{1}^{(n-2)}=d^{-1} f_{1,1} f_{3, n} ; \quad\left(a_{1}^{(n-2)}-b_{1}^{(n-2)}\right)^{-1}=f_{2} \\
a_{n-1}^{(n-2)}-b_{n-1}^{(n-2)}=w-D_{3}-\left(D_{1}-D_{3}\right)=w-D_{1}=f_{1} .
\end{array}\right.
$$

From (4.1)-(4.3) we obtain, by virtue of Definition 1,

$$
\begin{gathered}
a^{(n-1)}=f_{2}\left(d^{-1} f_{1} f_{3, n-1}, f_{1} f_{3, n-2}, \ldots, f_{1} f_{3}, f_{1}, 1\right) \\
a^{n-1}=\left(d^{-1} f_{1, n-1}, f_{1, n-2}, \ldots, f_{1,2}, f_{2}\right) .
\end{gathered}
$$

(4.4) is important. If we compare the latter with (2.7) we obtain

$$
a^{(0)} \stackrel{(d)}{=} a^{(n-1)}
$$

which means to say that $a^{(0)}=a^{(n-1)}$ in the case $d=1$. We have thus obtained the interesting

THEOREM 1. The ACF of the fixed vector $a^{(0)}$ from (2.7), with the notations (2.3) and (2.5), is purely periodic in the case $d=1$, and the length of the primitive period equals $m=n-1$.

The latter has the form

$$
\left\langle a^{(0)}, a^{(1)}, \ldots, a^{(i)}, \ldots, a^{(n-2)}\right\rangle, \quad i=2, \ldots, n-2 .
$$

$a^{(0)}$ from (2.7), $a^{(1)}$ from (2.11), $a^{(i)}$ from (3.5) $(i=2, \ldots, n-2)$, substituting in these formulas $d=1$. The corresponding companion vectors have the form:

$$
\text { (4.6) }\left\{\begin{array}{l}
b^{(0)}=\left(0,0, \ldots, 0, D_{1}-D_{2}\right), \\
b^{(1)}=\left(0,0, \ldots, 0, D_{1}-D_{n}\right), \ldots, \\
b^{(l)}=\left(0,0, \ldots, 0, D_{1}-D_{n-\imath+1}\right), \quad i=2, \ldots, n-2 .
\end{array}\right.
$$


The reader is reminded that while $d=1$ in Theorem 1 , the other $n$ elements $D_{1}, \ldots, D_{n}$ of the set $S_{n+1}$ may still be any algebraic numbers. It is surprising that in the case $d=1$ the ACF of the fixed vector $a^{(0)}$ from (2.7) indeed is purely periodic with the astonishingly short primitive period $m=n-1$. In the case $d \neq 1$, the ACF of the same $a^{(0)}$ is also purely periodic, but the length of its primitive period equals $m=n(n-1)$. This will be proved in the next sections. But already, we have learned from the previous section, that in the case $n=2$ the ACF of $a^{(0)}$ from (2.7) has primitive period of length $m=2=2(2-1)$ when $d \neq 1$, and $m=1=2-1$ when $d=1$; and in the case $n=3, m=6=3(3-2)$ when $d \neq 1$, and $m=2=3-1$ when $d=1$.

5. Periodicity of the ACF of $a^{(0)}$-completed. We shall use a new manner of writing the vectors of the primitive period of the ACF of $a^{(0)}$ from Theorem 1 (with $d=1$ ) and introduce

$$
g^{(i)}=\left(g_{1}^{(i)}, g_{2}^{(i)}, \ldots, g_{n-1}^{(i)}\right), \quad i=0,1, \ldots, n-2 ; n \geq 4 .
$$

It will be useful, for later purposes, to write out in full the values of $g_{j}^{(i)}$ from (2.7), (2.11), (3.5) where $d=1$,

$$
\left\{\begin{aligned}
g^{(0)}= & \left(g_{1}^{(0)}, g_{2}^{(0)}, \ldots, g_{n-1}^{(0)}\right)=\left(f_{1, n-1}, f_{1, n-2}, \ldots, f_{1,2}, f_{2}\right) \\
g^{(1)}= & \left(g_{1}^{(1)}, g_{2}^{(1)}, \ldots, g_{n-1}^{(1)}\right) \\
= & \left(f_{1, n-2} f_{n}, f_{1, n-3} f_{n}, \ldots, f_{1,2} f_{n}, f_{1} f_{n}, f_{n}\right) \\
g^{(i)}= & \left(g_{1}^{(i)}, g_{2}^{(i)}, \ldots, g_{n-1}^{(i)}\right) \\
= & \left(f_{1, n-i-1} f_{n-i+1, n}, f_{1, n-i-2} f_{n-i+1, n}, \ldots,\right. \\
& f_{1,2} f_{n-i+1, n}, f_{1} f_{n-i+1, n}, f_{1} f_{n-i+1, n-1}, \ldots, \\
& \left.f_{1} f_{n-i+1}, f_{n-i+1}\right) \\
i=2,3, \ldots, n-2 . &
\end{aligned}\right.
$$

With the notation (5.2) we shall now write out in full a few of the fugues of the ACF of $a^{(0)}$ from (2.7) with $d \neq 1$.

$$
\left\{\begin{array}{l}
a^{(0)}=\left(g_{1}^{(0)}, g_{2}^{(0)}, \ldots, g_{n-1}^{(0)}\right), \\
a^{(1)}=\left(d^{-1} g_{1}^{(1)}, d^{-1} g_{2}^{(1)}, \ldots, d^{-1} g_{n-1}^{(1)}\right), \\
a^{(2)}=\left(d^{-1} g_{1}^{(2)}, d^{-1} g_{2}^{(2)}, \ldots, d^{-1} g_{n-1}^{(2)}\right), \\
\vdots \\
a^{(n-2)}=\left(d^{-1} g_{1}^{(n-2)}, d^{-1} g_{2}^{(n-2)}, g_{3}^{(n-2)}, \ldots, g_{n-1}^{(n-2)}\right) .
\end{array}\right.
$$

(5.3) is the first fugue. 


$$
\left\{\begin{array}{l}
a^{(1(n-1)+0)}=\left(d^{-1} g_{1}^{(0)}, g_{2}^{(0)}, g_{3}^{(0)}, \ldots, g_{n-1}^{(0)}\right), \\
a^{(1(n-1)+1)}=\left(g_{1}^{(1)}, g_{2}^{(1)}, \ldots, g_{n-1}^{(1)}\right), \\
a^{(1(n-1)+2)}=\left(d^{-1} g_{1}^{(2)}, d^{-1} g_{2}^{(2)}, \ldots, d^{-1} g_{n-1}^{(2)}\right), \\
a^{(1(n-1)+3)}=\left(d^{-1} g_{1}^{(3)}, d^{-1} g_{2}^{(3)}, \ldots, d^{-1} g_{n-2}^{(3)}, g_{n-1}^{(3)}\right), \\
\vdots \\
a^{(1(n-1)+n-2)}=\left(d^{-1} g_{1}^{(n-2)}, d^{-1} g_{2}^{(n-2)}, d^{-1} g_{3}^{(n-2)}, g_{4}^{(n-2)}, \ldots, g_{n-1}^{(n-2)}\right) .
\end{array}\right.
$$

(5.4) is the second fugue.

$$
\left\{\begin{array}{l}
a^{(2(n-1)+0)}=\left(d^{-1} g_{1}^{(0)}, d^{-1} g_{2}^{(0)}, g_{3}^{(0)}, \ldots, g_{n-1}^{(0)}\right) \\
a^{(2(n-1)+1)}=\left(d^{-1} g_{1}^{(1)}, g_{2}^{(1)}, \ldots, g_{n-1}^{(1)}\right) \\
a^{(2(n-1)+2)}=\left(g_{1}^{(2)}, g_{2}^{(2)}, \ldots, g_{n-1}^{(2)}\right) \\
a^{(2(n-1)+3)}=\left(d^{-1} g_{1}^{(3)}, d^{-1} g_{2}^{(3)}, \ldots, d^{-1} g_{n-1}^{(3)}\right) \\
a^{(2(n-1)+4)}=\left(d^{-1} g_{1}^{(4)}, d^{-1} g_{2}^{(4)}, \ldots, d^{-1} g_{n-2}^{(4)}, g_{n-1}^{(4)}\right) \\
\vdots \\
a^{(2(n-1)+n-2)}=\left(d^{-1} g_{1}^{(n-2)}, \ldots, d^{-1} g_{4}^{(n-2)}, g_{5}^{(n-2)}, \ldots, g_{n-1}^{(n-2)}\right)
\end{array}\right.
$$

(5.5) is the third fugue.

The reader will now have no difficulty in proving

LEMMA 2. The factors $d^{-1}$ appearing before the $g_{i}^{(j)}$ in the current vectors of the $\mathrm{ACF}$ of $a^{(0)}$ from (2.7) with $d \neq 1$ appear subsequently one after the other starting with the first component of the vector; their frequency of occurrence in a vector is $k, 0 \leq k \leq n-1$; if in a vector the frequency of $d^{-1}$ is $k$, then in the next vector it is $k-1$; if its frequency in one vector is zero, then in the next vector, it is $n-1$.

For the $j$ th vector of the $i+1$ st fugue we shall use the notation

$$
a^{(i(n-1)+j)}=(k) g^{(j)}
$$

where $(k)$ denotes the frequency of the factor $d^{-1}$ in this vector $g^{(j)}$. Of course, (5.6) does not say how to state explicitly any such vector $a^{(i(n-1)+j)}$. For this purpose a functional relation between $i, j$ and $k$ is necessary and most important. This is:

$$
\begin{cases}i-j \equiv k(n), & k=0,1, \ldots, n-1 ; \\ \text { For } k<0, \text { take } n-k . & j=0,1, \ldots, n-2 .\end{cases}
$$

The proof of (5.7) is entirely based on Lemma 2. 
If in a certain fugue we have the vector $a^{i(n-i)+j}$, then in the next vector we have $j+1$, and since $k$ decreases by one in this next vector, it becomes $k-1$, so that $i-(j+1) \equiv k-1(n)$ or $i-j \equiv k(n)$, as should be. If we look for the next fugue with the same row-vector $j$, viz. $a^{(i+1)(n+1)+j}$, then the frequency of $k$ for the same $j$ is one greater, namely $k+1$, so that we have $(i+1)-j \equiv k+1(n)$ or again, $i-j \equiv k(n)$. This proves (5.7) by induction, since it is correct for $i=j=k=0$.

We are now able to write down any current vector in the ACF of $a^{(0)}$. For example, let $n=9, m=9 \cdot 8=72$, and we want to find $a^{(61)}=$ $a^{7(9-1)+5} ; i=7, j=5 ; 7-2=2=k$. Hence

$$
a^{(61)}=\left(d^{-1} g_{1}^{(5)}, d^{-1} g_{2}^{(5)}, g_{3}^{(5)}, \ldots, g_{8}^{(5)}\right) \text {. }
$$

We now ask the decisive question: Can ACF of $a^{(0)}$ become purely periodic, and what is the length $m$ of the primitive period? (Every periodic ACF can be transformed into a purely periodic ACF.) In such a case we must have

$$
j=k=0, \text { as in } a^{(0)} .
$$

(5.7), (5.8) result in chooseing $\min . i$,

$$
i \equiv 0(n), \quad i=n \text {. }
$$

We have obtained:

THEOREM 2. The ACF of the fixed vector $a^{(0)}$ from (2.7) with the notation (2.3), (2.5) and $d \neq 1$ is purely periodic and the length of its primitive period is $m=n(n-1)$, consisting of $n$ fugues.

The length of the primitive period of the ACF is indeed very large but, as it will be shown, it does not prevent us from calculating units.

6. Irreducibility and roots of polynomials. Bernstein [3, pp. 72-79] has proved the following result which we state here with some slight alterations:

THEOREM 3. Let

$$
\left\{\begin{array}{l}
P(x)=x^{n}+\left(\sum_{j=1}^{n-1} k_{j} x^{n-\jmath}\right)-d, \\
d, k_{1}, \ldots, k_{n-1} \in \mathbf{Z} ; \quad d \mid k_{j} \quad(j=1, \ldots, n-1), \\
\left|k_{n-1}\right| \geq 2|d|(2+B) ; \quad B=1+\sum_{j=1}^{n-2}\left|k_{j}\right| .
\end{array}\right.
$$

Then $P(x)$ is irreducible and has at least one real root. 
If $d$ is square free and $|d|>1$, then irreducibility of $P(x)$ follows from Eisenstein's Criterion. But this would then exclude $|d|=1$. Bernstein's theorem is valid also for any algebraic integers $d, k_{1}, \ldots, k_{n-1}$, $d \mid k_{n-1}$. This we could well use, but it would take us too long to prove it.

We introduce a polynomial which will be central to our investigation on units, viz.

$$
\left\{\begin{array}{l}
T(x)=-d+\prod_{i=1}^{k}\left(x^{s_{\imath}}-D_{i}^{s_{l}}\right), \\
d, D_{i} \in \mathbf{Z} ; \quad d \mid D_{i} ; \quad i=1,2, \ldots, k \\
s_{i} \geq 1 ; \quad \text { if all } s_{\imath}=1, \quad \text { then } k \geq 2 ; \\
0<D_{1}<D_{2}<\cdots<D_{k} .
\end{array}\right.
$$

We prove

LEMMA 3. $T(x)$ is irreducible in infinitely many cases and has, in these cases, at least one real root.

We rearrange (6.2):

$$
\left\{\begin{array}{l}
T(y)=-d+\prod_{i=1}^{k}\left[\left(y+D_{k}\right)^{s_{i}}-D_{i}^{s_{i}}\right], \\
y=x-D_{k} .
\end{array}\right.
$$

From (6.3)

$$
\left\{\begin{array}{l}
T(y)=-d+\left(y^{s_{k}}+\left(\begin{array}{c}
s_{k} \\
1
\end{array}\right) D_{k} y^{s_{k}-1}+\cdots+\left(\begin{array}{c}
s_{k} \\
s_{k}-1
\end{array}\right) D_{k}^{s_{k}-1} y\right) \\
\cdot \prod_{i=1}^{k-1}\left[\left(y+D_{k}\right)^{s_{i}}-D_{i}^{s_{i}}\right] \\
=y^{n}+t_{1} y^{n-1}+\cdots+t_{n-1} y-d, \\
d \mid t_{i} ; \quad t_{n-1}=\left(\begin{array}{c}
s_{k} \\
s_{k}-1
\end{array}\right) D_{k}^{s_{k}-1} \prod_{i=1}^{k-1}\left(D_{k}^{s_{i}}-D_{i}^{s_{i}}\right), \\
n=s_{1}+s_{2}+\cdots+s_{k} ; \quad i=1, \ldots, n-1 \text { in } t_{i},
\end{array}\right.
$$

where $d \mid t_{i}$ since all $t_{i}$ are polynomials in $D_{1}, D_{2}, \ldots, D_{k}$ with rational coefficients; further $t_{n-1}$ contains the highest power of $D_{k}$, viz. $s_{k}-1+$ $s_{1}+s_{2}+\cdots+s_{k-1}=n-1$. Hence we can choose sufficiently large $D_{k}$ so that $\left|t_{n-1}\right| \geq 2|d|\left(B_{1}+2\right), B_{1}=1+\sum_{j=1}^{n-2}\left|t_{j}\right|$. Thus the polynomial $T(y)$, hence also $T(x)$, satisfies the conditions of the polynomial $P(x)$, 
and Theorem 3, so that $T(x)$ is irreducible and has at least one real root in infinitely many cases.

In case $s_{k}=n, T(x)$ becomes

$$
\begin{array}{ll}
T(x)=x^{n}-D^{n}-d, d \mid D, n \geq 2 ; & D \geq 1 . \\
\left.T(y)=y^{n}+\sum_{j=1}^{n-1}\left(\begin{array}{l}
n \\
j
\end{array}\right) D^{j} y^{n-j}\right)-d ; & d, D \in \mathbf{Z},
\end{array}
$$

and we have to choose

$$
\left(\begin{array}{c}
n \\
1
\end{array}\right) D^{n-1} \geq 2|d|\left[3+\left(\begin{array}{l}
n \\
1
\end{array}\right) D+\left(\begin{array}{l}
n \\
2
\end{array}\right) D^{2}+\cdots+\left(\begin{array}{c}
n \\
n-2
\end{array}\right) D^{n-2}\right] .
$$

It suffices to choose $D \geq 2^{n+1}|d| ; n \geq 2$. Concluding, we shall write $T(x)$ in the form of $(2.1)$.

$$
\left\{\begin{array}{l}
G_{T}(x)=-d+\prod_{i=1}^{k} \prod_{j=1}^{s_{t}}\left(x-\rho_{j}^{j-1} D_{j}\right) \\
\rho_{j}=\exp \frac{2 \pi \sqrt{-1}}{s_{i}} \cdot \quad(\text { We write } \sqrt{-1} \text { to avoid } \\
\text { confusion with the index } i .)
\end{array}\right.
$$$$
\left\{\begin{array}{c}
\left\{D_{1}, \rho_{1} D_{1}, \ldots, \rho_{1}^{s_{1}-1} D_{1}, D_{2}, \rho_{2} D_{2}, \ldots, \rho_{2}^{s_{2}-1} D_{2}\right. \\
\left.\quad \ldots, D_{k}, \rho_{k} D_{k}, \ldots, \rho_{k}^{s_{k}-1} D_{k}\right\} \\
=\left\{\bar{D}_{1}, \bar{D}_{2}, \ldots, \bar{D}_{n}\right\}, \text { for useful notation. }
\end{array}\right.
$$

Let

$$
\left\{\begin{array}{l}
\left(s_{1}, s_{2}, \ldots, s_{k}\right)=s ; \quad \bar{\rho}=\exp \frac{2 \pi \sqrt{-1}}{s} \\
G_{T}(\bar{w})=0 ; \quad \bar{w} \text { real } ; \quad Q\left(\bar{D}_{1}, \bar{D}_{2}, \ldots, \bar{D}_{k}, \bar{w}\right)=Q(\bar{\rho}, w)
\end{array}\right.
$$

$$
G_{T}(x)=-d+\prod_{j=1}^{n}\left(x-\bar{D}_{j}\right) ; \quad-d+\prod_{j=1}^{n}\left(\bar{w}-\bar{D}_{j}\right)=0 .
$$

\section{Units. the main result.}

\section{DEFINITION 4. Let}

$$
\left\{\begin{array}{l}
\mathscr{K} \text { be an algebraic number field; } \\
a^{(0)}=\left(a_{1}^{(0)}, a_{2}^{(0)}, \ldots, a_{n-1}^{(0)}\right) \in \mathcal{K}^{n-1}, n \geq 2, \text { and } \\
a_{1}^{(0)}, a_{2}^{(0)}, \ldots, a_{n-1}^{(0)} \quad \text { algebraic integers. }
\end{array}\right.
$$

Then $a^{(0)}$ is called an integral vector. 
THEOREM 4. Let

$$
\left\{\begin{array}{l}
\mathcal{K}_{1}, \mathscr{K}_{2} \text { be algebraic number fields; } \\
\mathscr{K}_{1} \subset \mathcal{K}_{2} ; \quad\left[\mathcal{K}_{2}: \mathscr{K}_{1}\right]=g \geq n \geq 2 ; \\
a^{(0)} \in \mathscr{K}_{2} \text { an integral vector } ; \\
\left\langle b^{(v)}\right\rangle_{v=0}^{\infty} \text { a sequence of integral vectors } \in \mathscr{K}_{1} ; \\
A_{i}^{(v)} \text { the matrix entries from }(1.9) .
\end{array}\right.
$$

If the ACF of $a^{(0)}$, carried out with the companion vectors $b^{(v)}$ is purely periodic with $m=$ the length of the primitive period then the algebraic number

$$
\prod_{i=1}^{m} a_{n-1}^{(i)}=A_{0}^{(m)}+a_{1}^{(0)} A_{0}^{(m+1)}+\cdots+a_{n-1}^{(0)} A_{0}^{(m+n-1)}
$$

is a unit in $\mathcal{K}_{2}$ (relative to $\mathcal{K}_{1}$ ).

Proof. Since, because of periodicity, we have $a_{i}^{(m)}=a_{i}^{(0)}, i=1, \ldots$, $n-1$, we obtain (7.3) from (1.13). The $a_{i}^{(0)}$ are algebraic integers by hypothesis, so are the $A_{0}^{(m+j)}, j=0,1, \ldots, n-1$, since the $b^{(v)}$ are integral by hypothesis. Thus $\sum_{j=0}^{n-1} a_{j}^{(0)} A_{0}^{(m+j)}$ in (7.3) is an algebraic integer.

We further note that, by virtue of the conditions of the theorem, all entries in (1.14) are algebraic integers, so that $\left(\sum_{j=0}^{n-1} a_{j}^{(0)} A_{0}^{(m+j)}\right)^{-1}$ is an integer. This proves the theorem.

We now return to the vector $a^{(0)}$ from (2.7), substituting there $\bar{a}^{(0)}$ for $a^{(0)}, \bar{D}_{1}, \bar{D}_{2}, \ldots, \bar{D}_{n}$ for $D_{1}, D_{2}, \ldots, D_{n}$ from (6.6), and now let $d$ have the value from (6.2), viz. $d$ a rational integer. Theorem 2 then becomes: The ACF of the fixed vector $\bar{a}^{(0)}$ with $d \neq 1$ is purely periodic and the length of its primitive period $m=n(n-1)$, consisting of $n$ fugues. From (5.3)(5.7) we learn a few important facts about the ACF of $\bar{a}^{(0)}$ :

(i) for $D_{1}$ we substitute $\bar{D}_{1}$; altogether there are $n$ such choices of substitution;

(ii) the numbers $\bar{D}_{1}, \bar{D}_{2}, \ldots, \bar{D}_{n}$ are all algebraic integers, since $\bar{D}_{j}=$ $\rho_{j}^{t} D_{j},\left(t=0,1, \ldots, s_{j}-1\right)$, and $\rho_{j}$ and $D_{j}$ are integers;

(iii) $d \mid \bar{D}_{j}(j=1, \ldots, n-1)$, since $D \mid D_{j}$;

(iv) the product of the last components of $n-1$ of the $n$ fugues of the primitive period of ACF has the form:

$$
d^{-1} \prod_{j=2}^{n}\left(\bar{w}-\overline{D_{j}}\right)
$$


(v) One fugue out of the $n$ fugues does not have among its last components the factor $d^{-1}$. This fugue is that one whose first vector has $k=n-2$, so that

$$
i-0 \equiv k-2(n) ; \quad i=n-2 .
$$

Thus the product of the $n-1$ st $(i+1)$ fugue equals (with $i=n-2$ )

$$
\prod_{j=2}^{n}\left(\bar{w}-\bar{D}_{j}\right) \text {. }
$$

(vi) All the entries of the companion vectors of the $n$ fugues are either zero or $\bar{D}_{1}-\bar{D}_{j}$ or $d^{-1}\left(\bar{D}_{1}-\bar{D}_{j}\right), j=2, \ldots, n,(j \neq n-1)$. Hence they are all integers (and so are the $A_{i}^{(v)}$ ).

(vii) Because of (7.4), (7.5), we have

$$
\prod_{j=0}^{n(n-1)-1} \bar{a}_{n-1}^{(j)}=d^{-(n-1)}\left(\prod_{j=2}^{n}\left(\bar{w}-\bar{D}_{j}\right)^{n} .\right.
$$

But because of periodicity $a_{n-1}^{n(n-1)}=\bar{a}_{n-1}^{(0)}$, hence from (7.6)

$$
\prod_{j=0}^{n(n-1)-1} \bar{a}_{n-1}^{(j)}=\prod_{j=1}^{n(n-1)} \bar{a}_{n-1}^{(j)},
$$

so that, by virtue of (7.6), (7.7) and Theorem 4 , we have obtained the second main result in

THEOREM 5. With the meanings of $\bar{w}$ and $\bar{\rho}, \bar{D}_{j}$, as explained before,

$$
e=d^{-(n-1)}\left(\prod_{j=2}^{n}\left(\bar{w}-\bar{D}_{j}\right)\right)^{n}
$$

is a unit in $Q(\bar{w}, \bar{\rho})$.

8. Units from Theorem 5. The unit $e$ from (7.8) has a complicated form unsuitable to practicalities. We shall find simpler forms for units in $Q(\bar{w}, \bar{\rho})$, and also disclose units in $Q(\bar{w})$. Since we took $\bar{w}=w_{0}$ to be a real root of $G_{T}(x)$, we could have taken any of the roots $w_{0}, w_{1}, \ldots, w_{n-1}$ of $G_{T}(x)$ (some, even all of which may be real) and still have obtained the unit $d^{-(n-1)}\left(\Pi_{j=2}^{n}\left(\bar{w}_{t}-\bar{D}_{j}\right)\right)^{n}, t=0,1, \ldots, n-1$. Also, since $\bar{D}_{1}$ may assume any of the values

$$
\bar{D}_{1}=\rho_{i}^{u} D_{i}, \quad i=1, \ldots, n ; \quad u=0, \quad s_{1}, \ldots, s_{i}-1,
$$

we can obtain altogether $n$ units of the form (7.8) for $\bar{w}$ constant, $\bar{w}=w_{0}$, and altogether $n^{2}$ units in $Q\left(w_{t}, \bar{\rho}\right)$ among them some units in $Q(\bar{w}), \bar{w}$ 
real, as we shall soon see. From (6.8) we obtain

$$
\prod_{j=2}^{n}\left(\bar{w}-\bar{D}_{j}\right)=\frac{d}{\bar{w}-\bar{D}_{1}}
$$

Hence from (7.8), (8.2)

$$
\begin{gathered}
e=\frac{d}{\left(\bar{w}-\bar{D}_{1}\right)^{n}}, \\
\left\{\begin{array}{l}
e_{1}=\frac{\left(\bar{w}-\bar{D}_{1}\right)^{n}}{d}, \\
\text { is a unit in } Q\left(\bar{w}, \bar{D}_{1}\right) .
\end{array}\right.
\end{gathered}
$$

Since in (8.3) we can use any of the $\bar{D}_{j},(j=1, \ldots, n)$ instead of $\bar{D}_{1}$, we obtain

THEOREM 6. All the numbers

$$
e_{j}=\frac{\left(\bar{w}-\bar{D}_{j}\right)^{n}}{d}, \quad j=1, \ldots, n,
$$

are units in $Q(\bar{w}, \bar{\rho})$. Among them

$$
e^{(i)}=\frac{\left(\bar{w}-D_{i}\right)^{n}}{d}, \quad i=1, \ldots, k ; \quad k \text { from }(6.2)
$$

are units in the real field $Q(\bar{w})$.

The product

$$
\prod_{u=0}^{s_{t}-1}\left(\frac{\left(\bar{w}-\rho_{i}^{u} D_{i}\right)^{n}}{d}\right)=\frac{\left(\bar{w}^{s_{t}}-D_{i}^{s_{t}}\right)^{n}}{d^{s_{i}}}
$$

is a unit as a product of units. Hence

\section{COROLLARY 1. The numbers}

(8.6) $e^{\left(s_{t}\right)}=\frac{\left(\bar{w}^{s_{t}}-D_{i}^{s_{t}}\right)^{n}}{d^{s_{t}}}, \quad i=1,2, \ldots, k ; k$ from $(6.2), s_{i} \geq 1$

are units in the real field $Q(\bar{w})$.

From (8.5) we obtain

$$
\left(e^{(i)}\right)^{-s_{i}}=\frac{d^{s_{i}}}{\left[\left(\bar{w}-D_{i}\right)^{s_{i}}\right]^{n}},
$$


so that

$$
\left(e^{(i)}\right)^{-s_{i}} e^{\left(s_{t}\right)}=\left(\frac{\bar{w}^{s_{\iota}}-D_{i}^{s_{i}}}{\left(\bar{w}-D_{i}\right)^{s_{l}}}\right)^{n},
$$

is a unit, hence

COROLlaRY 2. The numbers

(8.7) $\quad e_{i i}=\frac{\bar{w}^{s_{i}}-D_{i}^{s_{i}}}{\left(\bar{w}-D_{i}\right)^{s_{i}}}, \quad i=1, \ldots, k ; \quad k$ from $(6.2), s_{i}>1$

are units in the real field $Q(\bar{w})$.

Let

$$
\left\{\begin{array}{l}
n=s_{i} t_{i}, \quad s_{i}, t_{i}>1 \\
\text { for some } i \leq k
\end{array}\right.
$$

Substituting $n$ from (8.8) in (8.6), we obtain

$$
e^{\left(s_{t}\right)}=\frac{\left[\left(\bar{w}^{s_{t}}-D_{i}^{s_{t}}\right)^{t_{t}}\right]^{s_{t}}}{d^{s_{t}}},
$$

hence

Corollary 3. Let $n=s_{l} t_{i}, s_{i}, t_{i}>1 ; s_{i} \in\left\{s_{1}, s_{2}, \ldots, s_{k}\right\}$. Then

$$
e^{\left(t_{i}\right)}=\frac{\left(\bar{w}^{s_{i}}-D_{t}^{s_{i}}\right)^{t_{i}}}{d}
$$

are units in the real field $Q(\bar{w})$.

Let $|d|=1$. Then we have

COROLlaRY 4. The numbers

$$
\text { (8.10) }\left\{\begin{array}{l}
e_{j, 1}=\bar{w}-\bar{D}_{j}, \quad u=1, \ldots, n \text { are units in } Q(\bar{w}, \bar{\rho}) . \\
e_{1}^{(i)}=\bar{w}-D_{i}, \quad i=1, \ldots, k \text { are units in the real field } \\
Q(\bar{w}), k \text { from }(6.2) \\
e_{1}^{\left(s_{i}\right)}=\bar{w}^{s_{i}}-D_{i}^{s_{1}}, \quad i=1, \ldots, k ; s_{\imath}>1 ; k \text { from }(6.2) \\
\text { are units in the real field } Q(\bar{w}) .
\end{array}\right.
$$


9. Derivation of previously known units. We return to the polynomial $T(x)$ of (6.2). In the case $k=1, T(x)$ takes the form, $s_{1}=n$,

$$
\left\{\begin{array}{l}
T(x)=x^{n}-D^{n}-d ; \quad d, D \in \mathbf{Z} ; D>0 ; n \geq 2 ; d \mid D \\
T(\bar{w})=0 ; \quad \bar{w} \text { real; } \bar{w}^{n}=D^{n}+d ; \bar{w} \text { of degree } n \text { over } Q .
\end{array}\right.
$$

But even in this case the method leading to the periodic ACF of $\bar{a}^{(0)}(w)$, and the derivation of units from it is the same. We obtain from (9.1)

$$
\left\{\begin{array}{l}
-d+\prod_{j=1}^{n}\left(\bar{w}-\bar{D}_{j}\right)=0 . \\
\overline{D_{J}}=\rho^{J-1} D ; \quad j=1, \ldots, n ; \bar{D}_{1}=D ; \\
\rho=\exp \frac{2 \pi \sqrt{-1}}{n} ; \quad d \mid \bar{D}_{J} \text { since } d \mid D .
\end{array}\right.
$$

With the conditions of (9.2) those of Theorems 4 and 5 are satisfied, and we have

THEOREM 7. Let conditions (9.1) and (9.2) hold. Then the n numbers

$$
e_{j}=\frac{\left(\bar{w}-\rho^{j-1} D\right)^{n}}{d} ; \quad j=1, \ldots, n
$$

are units in $Q(\bar{w}, \rho)$. The real number

$$
e_{1}=\frac{(\bar{w}-D)^{n}}{d}
$$

is a unit in the real field $Q(\bar{w})$.

$$
\text { If }|d|=1
$$

$$
e_{11}=\bar{w}-D \text { is a unit in } Q(\bar{w})
$$

Let $s t=n, s>1$. We obtain from $(9.1)$

$$
\left(\bar{w}^{s}\right)^{t}=\left(D^{s}\right)^{t}+d .
$$

Since $d \mid D$, we also have $d \mid D^{s}$, and all that was said in Theorem 7 about $\bar{w}, D$.

$$
e_{1}^{(s)}=\frac{\left(\bar{w}^{s}-D^{s}\right)^{t}}{d}
$$


is a unit in $Q(\bar{w})$. From (9.4), (9.6) we obtain

$$
e_{1}^{(s)} e_{1}^{-1}=\frac{\left(\bar{w}^{s}-D^{s}\right)^{t}}{d} \cdot \frac{d}{\left[(\bar{w}-D)^{s}\right]^{t}}=\left(\frac{\bar{w}^{s}-D^{s}}{(\bar{w}-D)^{s}}\right)^{t},
$$

a unit in $Q(w)$. Hence

COROllary 5. The $\tau(n)-1$ numbers

$$
e_{1}^{(s, t)}=\frac{\bar{w}^{s}-D^{s}}{(\bar{w}-D)^{s}}, \quad s t=n, s>1,
$$

are units in $Q(\bar{w})$.

If $n=p^{v}, p$ prime, $v=1,2, \ldots$, then instead of $d$, we have $p d$ which makes $d \mid D$ valid.

Bernstein and Hasse [5] obtained the units (9.4), (9.8) with the additional restrictions $D \geq(n-2) d$ for $d>0$ and $D \geq 2 d(n-1)$ for $d<0 ; n \geq 2$. These were the restrictions Bernstein $[1,2]$ needed for the periodicity of the Jacobi-Perron Algorithm with $w=\sqrt[n]{D^{n} \pm d}, d, D>0$, $d \mid D$.

In his most remarkable Ph.D. thesis Stender [13] proved that in the cases $n=3,4,6$ the possible 1, 2, 3 units from (9.4), (9.8) form a system of fundamental units in the respective fields. This was a breakthrough, but did not prove in general that the units gained from (periodic) JacobiPerron Algorithms are always fundamental.

The units are independent, whether they are fundamental or not, has not been proved for the general case. This fact is also true for the units obtained from ACF.

Later, Halter-Koch and Stender [8] proved that formulas (9.4), (9.8) hold for a much wider class of units.

Another breakthrough in units of this kind came with an important paper by Halter-Koch [7] who started with the polynomial

$$
\begin{aligned}
& G_{T}(x)=\prod_{i=1}^{r_{1}}\left(x-D_{i}\right) \prod_{j=r_{1}+1}^{r_{1}+r_{2}}\left(x-D_{j}\right)\left(x-\bar{D}_{j}\right)-d, \\
& r_{1} \geq 0, \quad r_{2} \geq 0, \quad n=r_{1}+2 r_{2}, \quad d, D_{i} \in Z ; \\
& D_{1}>D_{2}>\cdots>D_{r_{1}} ; \quad D_{j}, \bar{D}_{j} \text { complex conjugate, } \\
& d \text { a divisor of pairs of } D_{i} \text { and the } D_{i}, \\
& D_{i} \text { and } D_{j}, D_{j} \text { and } \bar{D}_{j} .
\end{aligned}
$$


Halter-Koch proves that $G_{T}(x)$ has exactly $r_{1}$ real roots. If $w$ is one of them then

$$
d^{-1}\left(w-D_{i}\right)^{n} \text { and } d^{-2}\left[\left(w-D_{j}\right)\left(w-\bar{D}_{j}\right)\right]^{n}
$$

are units in $Q(w)$. Of course these units can also be obtained from our ACF of $\bar{a}^{(0)}$. Bernstein [4] used his so-called zero algorithm to gain these Halter-Koch units.

The zero algorithm (abbr. ZA) is also a modification of JPA, and it is different from ACF.

The author conjectures that many more known units can be calculated by ACF. This will be discussed in a further paper.

\section{REFERENCES}

[1] L. Bernstein, Periodical continued fractions for irrationals of degree $n$ by Jacobi's algorithm, J. Reine, Angew. Math., 213 (1963), 31-38.

Representation of $\sqrt[n]{D^{n}-d}$ as a periodic continued fraction by Jacobi's algorithm, Math. Nachr., 29 (1965), 179-200.

[3] _ The Jacobi-Perron Algorithm, its Theory and Applications, Springer, BerlinHeidelberg-New York. Lect. Notes. Math., 207 (1971).

[4] _ An algorithm for Halter-Koch units, Michigan Math. J., 25 (1978), 371-377.

[5] L. Bernstein and H. Hasse, Einheitenberechnung mittels des Jacobi-Perronschen Algorithmus, J. Reine. Angew. Math., 218 (1965), 51-69.

[6] E. Dubois and R. Paysant-Le-Roux, Algorithme de Jacobi-Perron dans le extensions cubiques, C. R. Acad. Sci. Paris, Sér. A, 280 (1975), 183-186.

[7] F. Halter-Koch, Unabhängige Einheitensysteme für eine allgemeine Klasse algebraischer Zahlcörper, Abh. Math. Sem. Univ. Hamburg, 43 (1975), 85-91.

[8] F. Halter-Koch and J. Stender, Unabhängige Einheiten für die Korper $Q\left(\sqrt[n]{D^{n}+d}\right)$ mit d $\mid D^{n}$, Abh. Math. Sem. Univ. Hamburg, 42 (1974), 33-40.

[9] M. Neubrand, Einheiten in algebraischen Funktionen-und Zahlkörpern, J. Reine. Angew. Math., 303/304 (1978), 170-204.

[10] __ Scharen quadratischer Zahlkörper mit gleichgebauten Einheiten, Acta Arithmetica, (1981), 125-132.

[11] O. Perron, Grundlagen für eine Theorie des Jacobischen Kettenbruchalgorithmus, Math. Ann., 64 (1907), 1-76.

[12] _ Der Jacobische Kettenbruchalgorithmus in einem kubischen Zahlkörper I and II, Sitzungsber, Bayer. Akad. Wiss. Math.-Nat. K1. (1973), 9-22.

[13] H. J. Stender, Einheiten für eine allgemeine Klasse total reeller algebraischer Zahlörper, J. Reine Angew. Math., (1972), 151-178.

[14] Lösbare Gleichungen $a x^{n}-b y^{n}=c$ und Grundeinheiten für einige algebraische Zahlkörper vom Grade $n=3$, 4, 6, J. Reine. Angew. Math., 290 (1977), $24-62$.

Received February 23, 1982 and in revised form August 27, 1982. This paper is based on the author's Ph.D. dissertation written at the University of Houston, under the guidance of Professor Dr. Jürgen Schmidt. 


\section{PACIFIC JOURNAL OF MATHEMATICS \\ EDITORS}

DONALD BABBITT (Managing Editor)

University of California

Los Angeles, CA 90024

Hugo RossI

University of Utah

Salt Lake City, UT 84112

C. C. MOOre and ARThur OGus

University of California

Berkeley, CA 94720
J. Dugundi

Department of Mathematics

University of Southern California

Los Angeles, CA 90089-1113

R. FINN and H. SAMELSON

Stanford University

Stanford, CA 94305

ASSOCIATE EDITORS

$\begin{array}{lllll}\text { R. Arens } & \text { E. F. Beckenbach } & \text { B. H. Neumann } & \text { F. Wolf } & \text { K. Yoshida }\end{array}$ $(1906-1982)$

\section{SUPPORTING INSTITUTIONS}

UNIVERSITY OF ARIZONA

UNIVERSITY OF BRITISH COLUMBIA

CALIFORNIA INSTITUTE OF TECHNOLOGY

UNIVERSITY OF CALIFORNIA

MONTANA STATE UNIVERSITY

UNIVERSITY OF NEVADA, RENO

NEW MEXICO STATE UNIVERSITY

OREGON STATE UNIVERSITY
UNIVERSITY OF OREGON

UNIVERSITY OF SOUTHERN CALIFORNIA

STANFORD UNIVERSITY

UNIVERSITY OF HAWAII

UNIVERSITY OF TOKYO

UNIVERSITY OF UTAH

WASHINGTON STATE UNIVERSITY

UNIVERSITY OF WASHINGTON 


\section{Pacific Journal of Mathematics}

\section{Vol. 110, No. $1 \quad$ September, 1984}

Wojciech Abramczuk, A class of surjective convolution operators $\ldots \ldots \ldots \ldots 1$

K. Adachi, Extending bounded holomorphic functions from certain

subvarieties of a weakly pseudoconvex domain $\ldots \ldots \ldots \ldots \ldots \ldots$

Malvina Florica Baica, An algorithm in a complex field and its application

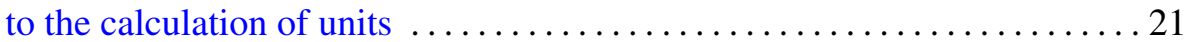

Giuliana Bianchi and Robert Cori, Colorings of hypermaps and a

conjecture of Brenner and Lyndon $\ldots \ldots \ldots \ldots \ldots \ldots \ldots \ldots \ldots \ldots \ldots \ldots$

Ronald James Evans, Determinations of Jacobsthal sums . . .......... 49

Leslie Foged, Characterizations of $\aleph$-spaces .................... 59

Nassif A. Ghoussoub and Paulette Saab, Weak compactness in spaces of

Bochner integrable functions and the Radon-Nikodým property . . . . . . 65

J. Gómez Gil, On local convexity of bounded weak topologies on Banach

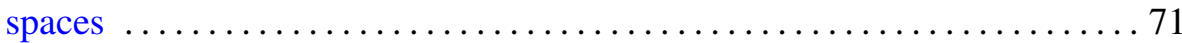

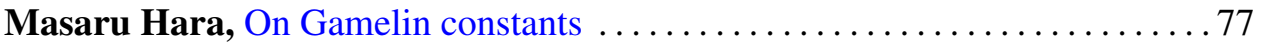

Wilfried Hauenschild, Eberhard Kaniuth and Ajay Kumar, Harmonic

analysis on central hypergroups and induced representations $\ldots \ldots \ldots 83$

Eugenio Hernandez, An interpolation theorem for analytic families of

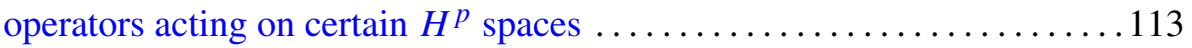

Thomas Alan Keagy, On "Tauberian theorems via block-dominated

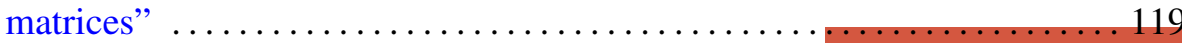

Thomas Landes, Permanence properties of normal structure $\ldots \ldots \ldots \ldots \ldots 125$

Daniel Henry Luecking, Closed ranged restriction operators on weighted

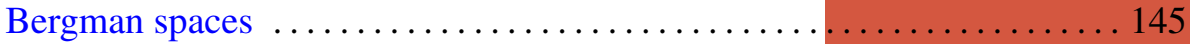

Albert Thomas Lundell, The $p$-equivalence of $\mathrm{SO}(2 n+1)$ and $\mathrm{Sp}(n) \ldots \ldots 161$

Mark D. Meyerson, Remarks on Fenn's "the table theorem" and Zaks' "the chair theorem" ..................................... 167

Marvin Victor Mielke, Homotopically trivial toposes . . . . . . . . . . 171

Gerard J. Murphy, Hyperinvariant subspaces and the topology on Lat A . . 183

Subhashis Nag, On the holomorphy of maps from a complex to a real manifold

Edgar Milan Palmer and Robert William Robinson, Enumeration of self-dual configurations ................................. 203

John J. Walsh and David Clifford Wilson, Continuous decompositions

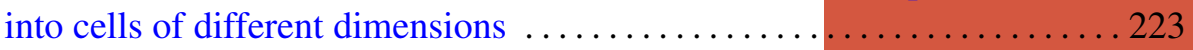

Walter John Whiteley, Infinitesimal motions of a bipartite framework .....233 